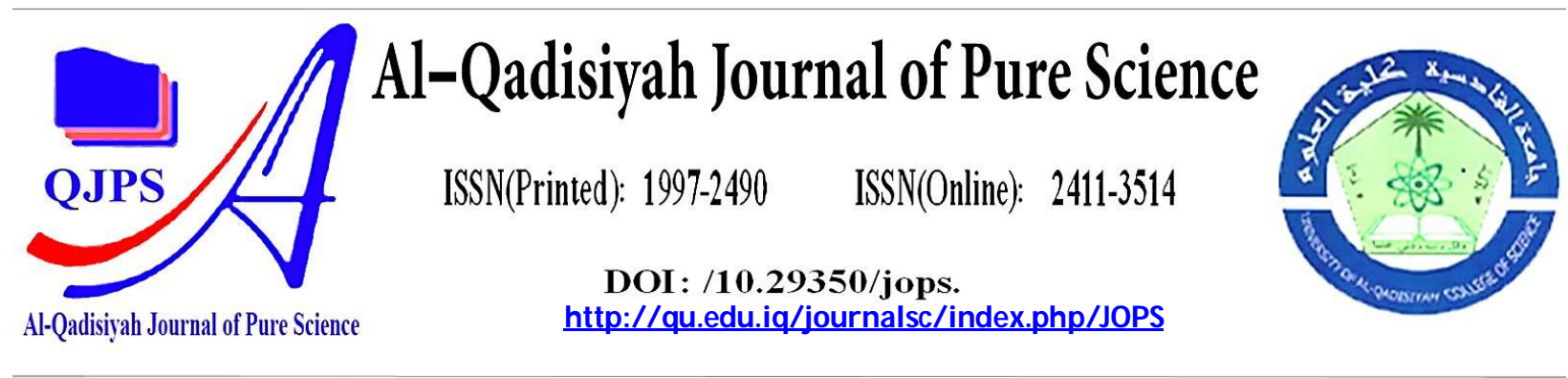

\title{
On s-g-cocompact open set and Continuity
}

\section{Authors Names \\ a. Raad Aziz Hussain Al- \\ Abdulla \\ b. Salam Satar Jabar \\ Article History \\ Received on: $27 / 03 / 2020$ \\ Revised on: 14/04/ 2020 \\ Accepted on: 05/ 05/ 2020

\begin{abstract}
Throughout this paper by a space we mean a supra topological space, we
\end{abstract} have studied some of properties to new set is called supra generalized- cocompact open set ( $\mathfrak{s - g - c o c - o p e n ~ s e t ) a n d ~ f i n d ~ t h e ~ r e l a t i o n ~ w i t h ~ o t h e r ~ s e t s ~}$ and our concluded a new class of the function called $\mathfrak{s}$-g-coc-continuous, $\mathfrak{s}$ - $g$ - coc'-continuous. We shall provide some properties of these concepts and it will explain the relationship among them and some results on this subjects are proved. Throughout this work, and new concept have been illustrated including, $\mathfrak{s - g - c o c - c o m p a c t ~ s p a c e ~ . ~}$}

Keywords: $\mathfrak{s - g - c o c - o p e n , ~} \mathfrak{s - g}$ coc-closed set, 5 -g-coc-

continuous, s-g-COC'-

continuous, $\mathfrak{s - g - c o c - c o m p a c t ~}$

space.

DOI: https://doi.org/10.29350/

jops.2020.25. 2.1104

\section{MSC: 30C45, 30C50}




\section{Preliminaries}

Definition(2.1) [3] If $X$ belongs to $\mu$ and $\mu$ is closed under arbitrary union, a subcollection $\mu$ of $2^{X}$ is considered a supra topology on $\mathrm{X}$. That $\mu$ elements is referred to as a supra open sets (5-open) of $(\mathrm{X}, \mu)$ and its complement is referred to as supra closed sets ( $\mathfrak{s}$-closed). The supra closure of $\mathrm{A}$ (denoted by $\bar{A}^{\mathfrak{s}}$ ) is the intersection of all supra closed sets containing $\mathrm{A}$ and the supra interior of $\mathrm{A}$ (denoted by $\mathrm{A}^{\circ \mathrm{s}}$ ) is the union of all supra open sets contained in A.The supra relative topology $\mu \mathrm{y}$ on $\mathrm{Y}$ is defined as $\mu \mathrm{y}=\{\mathrm{Y} \cap \mathrm{G}: \mathrm{G} \in \mu\}$. A pair $(\mathrm{Y}, \mu \mathrm{y})$ is called supra subspace of $(X, \mu)$.

Definition(2.2) [2]Let $(X, \mu)$ be a supra topological space. A subset A of $X$ is called supra g-closed (5-g-closed ) if $\bar{A}^{\mathfrak{s}} \subseteq \mathrm{U}$ whenever $\mathrm{A} \subseteq \mathrm{U}$ and $\mathrm{U}$ is supra open in $(\mathrm{X}, \mu)$. And $\mathrm{A}$ is called supra g-open ( $\mathfrak{s}$-g-open ), if $\mathrm{A}^{\mathrm{c}}$ is supra $\mathrm{g}$ closed.

$\operatorname{Remark}(2.3)$ [2] Every $\mathfrak{5}$-closed set implies $\mathfrak{5}$-g-closed set, $\mathfrak{5}$-g-closed set need not imply $\mathfrak{s}$-closed sets, $\mathfrak{5}$-open set mplies $\mathfrak{s}$-g-open and $\mathfrak{s}$-g-open must not imply $\mathfrak{s}$-open set .

Theorem(2.4) [2] A subset of supra topological space in $(X, \mu)$ is $\mathfrak{s}$-g-open set if and only if $B \subseteq A^{\circ \mathfrak{s}}$ whenever $B$ is $\mathfrak{s}$-closed and $\mathrm{B} \subseteq \mathrm{A}$.

Definition (2.5) [1] A supra topological spaces $(X, \mu)$ is called supra compact ( $\mathfrak{5}$-compact for short) provided that every supra open cover of $\mathrm{X}$ has a finite subcover .

Definition (2.6) [1]. A collection $\{\mathrm{Gi}: \mathrm{i} \in \mathrm{I}\}$ of supra open sets in a supra topological spaces $(\mathrm{X}, \mu)$ is called supra open cover of a subset $\mathrm{E}$ of $\mathrm{X}$ provided that $\mathrm{E} \subseteq \mathrm{U}\{\mathrm{Gi}: \mathrm{i} \in \mathrm{I}\}$.

\section{On supra generalized co-compact-open set and supra generalized co-compact -continuous} functions

In this section, we introduce the definition of $\mathfrak{s}$-g-coc-open set, $\mathfrak{s}$-g-coc-closed set , $\mathfrak{5}$-g-coc-continuous , $\mathfrak{s}$-gcoc'-continuous, remarks and propositions about this concept .

Definition (3.1) A subset A of a space $(X \mu)$ is called supra ganeralized cocompact open set (s-g-coc-open set) if for every $\mathrm{x} \in \mathrm{A}$ there exists $\mathfrak{s}$-g-open set $\mathrm{U} \subseteq \mathrm{X}$ and $\mathfrak{s}$-compact subset $\mathrm{K}$ such that $\mathrm{x} \in \mathrm{U}-\mathrm{K} \subseteq \mathrm{A}$, the complement of $\mathfrak{s}$-g-coc-open set is called $\mathfrak{s}$-g-coc-closed set. The family of all $\mathfrak{s}$-g-coc-open subsets of a space $(X, \mu)$ will be denoted by $\mu^{\mathrm{gk}}$.

Example(3.4) Let $X=\{a, b, c\}$ and $\mu=\{\varnothing, X,\{a, b\},\{a, c\}\}$. Then $\mu^{\mathrm{gk}}=p(X)$

\section{Proposition (3.5)}

i- Every $\mathfrak{s}$-open set is $\mathfrak{s}$-g-coc-open set.

iii-Every $\mathfrak{s}$-g-open set is $\mathfrak{s}$-g-coc-open set.

proof

i. Let $A$ s-open set Then $A$ s-g-open and let $K$ s-compact $(\varnothing)$. Then for all $x \in A$ we have $x \in U-K \subseteq A$ 
ii. Let $\mathrm{A}$ s-g-open and $\mathrm{K}$ s-compact $(\varnothing)$. Then for all $\mathrm{x} \in \mathrm{A}$ we have $\mathrm{x} \in \mathrm{U}-\mathrm{K} \subseteq \mathrm{A}$

but the convers of $\mathrm{i}$ and ii are not true for the following example :-

\section{Example (3.6)}

Let $X=N . \mu=\{\varnothing \cdot X X-\{1\}\}$ is supra topological space. It is clear that $\{1\} \mathfrak{s}$-g-coc-open set but not $\mathfrak{s}$-open and not $\mathfrak{s}$-g-open set .

Remark (3.7) :- Every $\mathfrak{5}$-g-closed is $\mathfrak{5}$-g-coc-closed but the converse is not true as in the example (3.6) .It is clear that $\{1\}^{\mathrm{c}}$ s-g-coc-closed set but $\{1\}^{\mathrm{c}}$ not $\mathfrak{s}$-g-closed set .

Proposition (3.8) :- The union of $\mathfrak{s}$-g-coc-open is $\mathfrak{s}$-g-coc-open set

Proof

let $\left\{A_{\ltimes}: \ltimes \in \Lambda\right\}$ s-g-coc-open sets . let $x \in A_{\ltimes}$.Then for some $\ltimes \in \wedge$. Then there exists $U_{\ltimes} \mathfrak{s}$-g-open set and $K_{\ltimes}$ s-compact such that $\mathrm{x} \in \mathrm{U}_{\ltimes}-\mathrm{K}_{\ltimes} \subseteq \mathrm{A}_{\ltimes} \subseteq \cup \mathrm{A}_{\ltimes}$ then $\mathrm{x} \in \mathrm{U}_{\ltimes}-\mathrm{K}_{\ltimes} \subseteq U \mathrm{~A}_{\ltimes}$, then $\cup \mathrm{A}_{\ltimes}$ s-g-coc-open set .

Theorem (3.9) :- $\mu^{\text {gk }}$ forms supra topology on X.

Proof.

By the definition one has directly that $\varnothing \in \mu^{\text {gk }}$.To see that $X \in \mu^{\text {gk }}$, let $\mathrm{x} \in \mathrm{X}$, take $\mathrm{U}=\mathrm{X}$ and $\mathrm{K}=\emptyset$. Then $\mathrm{x} \in \mathrm{U}-\mathrm{K}$ $\subseteq \mathrm{X}$

Let $\{U \alpha: \alpha \in \Delta\}$ be a collection of $\mathfrak{s}$-g-coc-open subsets of $(X, \mu)$ and $x \in U \alpha \in \Delta U \alpha$. Then there exists $\alpha 0 \in \Delta$ such that $\mathrm{x} \in \mathrm{U} \alpha^{\circ}$. Since $\mathrm{U} \alpha^{\circ}$ is $\mathfrak{s}$-g-coc-open, then there exists an $\mathfrak{s}$-g-open set $\mathrm{V}$ and $\mathfrak{s}$-compact subset $\mathrm{K}$, such that $\mathrm{x} \in \mathrm{V}-\mathrm{K} \subseteq \mathrm{U} \alpha^{\circ}$. Therefore, we have $\mathrm{x} \in \mathrm{V}-\mathrm{K} \subseteq \mathrm{U} \alpha^{\circ} \subseteq \mathrm{U} \alpha \in \Delta \mathrm{U} \alpha$.Hence, $\mathrm{U} \alpha \in \Delta \mathrm{U} \alpha$ is $\mathfrak{s}$-g-coc-open.

The following diagram shows the relation between types of $\mathfrak{s - c o c - o p e n ~ s e t s ~}$

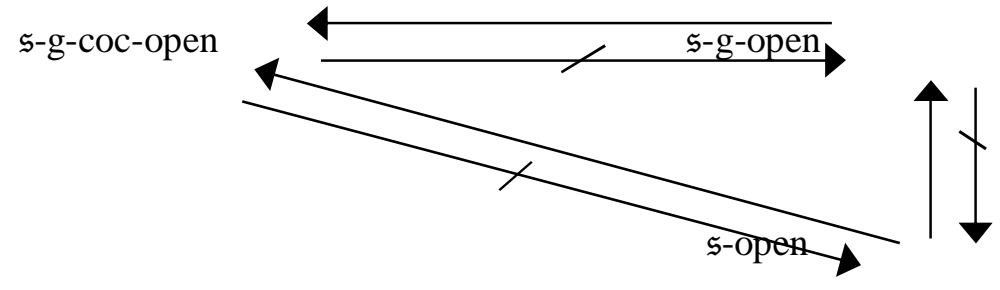

Definition (3.10) Let $X$ be a space and $A \subseteq X$. The intersection of all s-g-coc-closed sets of $X$ containing $A$ is called the s-g-coc-closure of A defined by .

$$
\bar{A}^{\mathfrak{s}-g-c o c}=\cap\{\mathrm{B}: \mathrm{B} \mathfrak{s}-\mathrm{g}-\text { coc-closed in } \mathrm{X} \text { and } \mathrm{A} \subseteq \mathrm{B}\}
$$

Proposition (3.11) Let $\mathrm{X}$ be a space and $\mathrm{A} \subseteq \mathrm{X}$, then $\mathrm{x} \in \bar{A}^{\mathfrak{s}-g-\operatorname{coc}}$ if and only if for each $\mathfrak{s}$-g-coc-open in $\mathrm{X}$ contained point $\mathrm{X}$ we have $\mathrm{U} \cap \mathrm{A} \neq \varnothing$.

\section{Proof :}

Asume that $\mathrm{x} \in \bar{A}^{\mathfrak{s}-g-c o c}$ and let $\mathrm{U} \mathfrak{s}$-g-coc-open in $\mathrm{X}$, such that $\mathrm{x} \in \mathrm{U}$, and suppose $\mathrm{U} \cap \mathrm{A}=\varnothing$, then $\mathrm{A} \subseteq \mathrm{U}^{\mathrm{c}}$ since $\mathrm{U}$ is $\mathfrak{s}$-g-coc-open set in $\mathrm{X}, \mathrm{x} \in \mathrm{U}$, then $\mathrm{U}^{\mathrm{c}}$ is $\mathfrak{s}$-g-coc-closed set in $\mathrm{X}, \mathrm{x} \notin \mathrm{U}^{\mathrm{c}}$, and $\bar{A}^{\mathfrak{s}-g-c o c}$ is smallest $\mathfrak{s}$-g-coc- 
closed set contain $\mathrm{A}$, then $\bar{A}^{\mathfrak{s}-g-c o c} \subseteq \mathrm{U}^{\mathrm{c}}$, since $\mathrm{U} \cap \mathrm{U}^{\mathrm{c}}=\emptyset \mathrm{x} \in \mathrm{U}$ then $\mathrm{x} \notin \mathrm{U}^{\mathrm{c}}$, then $\mathrm{x} \notin \bar{A}^{\mathfrak{s}-g-c o c}$, and this contradiction, thus $\forall$ s-g-open $U$ containing $\mathrm{x}$ we have $\mathrm{U} \cap \mathrm{A} \neq \emptyset$.

Conversely; Let $\mathrm{U}$ is $\mathfrak{s}$-g- coc-open set in $\mathrm{X}$, such that $\mathrm{x} \in \mathrm{U}$ an $\mathrm{U} \cap \mathrm{A} \neq \varnothing$. To prove $\mathrm{x} \in \bar{A}^{\mathfrak{s}-g-\operatorname{coc}}$, let $\mathrm{x} \notin$ $\bar{A}^{\mathfrak{s}-g-c o c}$, then $\mathrm{x} \in\left(\bar{A}^{\mathfrak{s}-g-c o c}\right)^{\mathrm{c}}$, since $\bar{A}^{\mathfrak{s}-g-c o c}$ is $\mathfrak{s}$-g-coc-closed, then $\left(\bar{A}^{s-g-c o c}\right)^{\mathrm{c}}$ is $\mathfrak{s - g}$-coc-open set in $\mathrm{X}$, and $\bar{A}^{\mathfrak{s}-g-c o c} \cap\left(\bar{A}^{\mathfrak{s}-g-c o c}\right)^{\mathrm{c}}=\varnothing$, which is contradiction since $\mathrm{A} \cap \mathrm{U} \neq \emptyset, \forall \mathrm{U}$ is $\mathfrak{s}$-g-coc-open set in $\mathrm{X}$, such that $\mathrm{x} \in \mathrm{U}$.

\section{Proposition (3.12)}

Let $\mathrm{X}$ be a space and $\mathrm{A} \subseteq \mathrm{B} \subseteq \mathrm{X}$ then

1. $\bar{A}^{\mathfrak{s}-g-\operatorname{coc}}$ is $\mathfrak{s - g}$-coc-closed set

2. A is $\mathfrak{s}$-g-coc-closed if and only if $\mathrm{A}=\bar{A}^{\mathfrak{s}-g-c o c}$

3. $\bar{A}^{\mathfrak{s}-g-c o c}=\overline{\bar{A}}^{\mathfrak{s}-g-\operatorname{coc}}{ }^{\mathfrak{s}-\mathrm{g}-\mathrm{coc}}$

4. If $\mathrm{A} \subseteq \mathrm{B}$ then $\bar{A}^{\mathfrak{s}-g-c o c} \subseteq \bar{B}^{\mathfrak{s}-g-\operatorname{coc}}$

5. $\bar{A}^{\mathfrak{s}-g-c o c} \subseteq \bar{A}^{\mathfrak{s}}$

\section{Proof :-}

1. By Proposition (3.8) .

2. Let $\mathrm{A}$ be $\mathfrak{s}$-g-coc-closed in $\mathrm{X}$. Since $\mathrm{A} \subseteq \bar{A}^{\mathfrak{s}-g-c o c}$ and $\bar{A}^{\mathfrak{s}-g-c o c}$ smallest $\mathfrak{s}$-g-coc-closed set containing $\mathrm{A}$, then $\bar{A}^{\mathfrak{s}-g-\operatorname{coc}} \subseteq \mathrm{A}$ then $\mathrm{A}=\bar{A}^{\mathfrak{s}-g-c o c}$

conversely :- Let $\mathrm{A}=\bar{A}^{\mathfrak{s}-g-c o c}$. Since $\bar{A}^{\mathfrak{s}-g-c o c}$ is $\mathfrak{s}$-g-coc-closed then A is $\mathfrak{s}$-g-coc-closed.

3. From (1) and (2)

4. Let $\mathrm{A} \subseteq \mathrm{B}$. Since $\mathrm{B} \subseteq \bar{B}^{\mathfrak{s}-g-c o c}$ then $\mathrm{A} \subseteq \bar{B}^{\mathfrak{s}-g-c o c}$ Since $\bar{B}^{\mathfrak{s}-g-c o c}$ smallest $\mathfrak{s - g}$-coc-closed set containing A then $\bar{A}^{\mathfrak{s}-g-c o c} \subseteq \bar{B}^{\mathfrak{s}-g-c o c}$ (since $\bar{A}^{\mathfrak{s}-g-c o c}$ smallest $\mathfrak{s}$-g-coc-closed set containing A).

5. Let $\mathrm{x} \in \bar{A}^{\mathfrak{s}-g-c o c}$ then for all $\mathfrak{s}$-g-coc-open set $\mathrm{U}$, such that $\mathrm{x} \in \mathrm{U}$ we have $\mathrm{U} \cap \mathrm{A} \neq \varnothing$. Then for all $\mathfrak{s}$-open set $\mathrm{U}$, such that $\mathrm{x} \in \mathrm{U}$, we have $\mathrm{A} \cap \mathrm{U} \neq \varnothing$ by proposition (3.11). Then $\mathrm{x} \in \bar{A}^{\mathfrak{s}}$.

Definition (3.13) Let $X$ be a space and $A \subseteq X$ The union of all $\mathfrak{s - g - c o c - o p e n ~ s e t s ~ o f ~} X$ containing in $A$ is called $\mathfrak{s}$ g-coc-Interior of A denoted by $\quad A^{\circ \mathfrak{s}-\mathrm{g}-\operatorname{coc}}$ or $\mathfrak{s}$-g-coc-In $(\mathrm{A})$

$$
\mathrm{A}^{\circ \mathfrak{s}-\mathrm{g}-\operatorname{coc}}=\cup\{\mathrm{B}: \mathrm{B} \mathfrak{s}-\mathrm{g}-\operatorname{coc}-\text { open in Xand } \mathrm{B} \subseteq \mathrm{A}\}
$$

Proposition (3.14) Let $X$ be a space and $A \subseteq X$, then $x \in A^{\circ \mathfrak{5}-g-c o c}$ if and only if there exists $\mathfrak{s}$-g-coc- open set $V$ containing $\mathrm{x}$ such that $\mathrm{x} \in \mathrm{V} \subseteq \mathrm{A}$.

Proof :

Let $x \in A^{\circ \mathfrak{s}-g-\operatorname{coc}}$ then $x \in U V$ such that $V$ s-g-coc-open set and $x \in V \subseteq A$.

Conversely; Let there exists $V$ s-g-coc-open set, such that $x \in V \subseteq A$ then $x \in U V, V \subseteq A$ and $V$ s-g-coc-open set ,then $x \in \mathrm{A}^{\circ \mathrm{os}-\mathrm{g}-\operatorname{coc}}$.

\section{Proposition (3.15)}

Let $X$ be a space and $A \subseteq B \subseteq X$, then .

1. $\mathrm{A}^{\circ \mathfrak{s}-\mathrm{g}-\mathrm{coc}}$ is $\mathfrak{s}$-g-coc-open set .

2. A is $\mathfrak{s - g - c o c - o p e n ~ i f ~ a n d ~ o n l y ~ i f ~} A=A^{\circ \mathfrak{s}-g-c o c}$.

3. $\mathrm{A}^{\circ \mathfrak{s}} \subseteq \mathrm{A}^{\circ \mathfrak{s}-\mathrm{g}-\mathrm{coc}}$. 
4. $\mathrm{A}^{\mathrm{os}-\mathrm{g}-\mathrm{coc}}=\left(\mathrm{A}^{\mathrm{os}-\mathrm{g}-\mathrm{coc}}\right)^{\mathrm{os}-\mathrm{g}-\mathrm{coc}}$.

5. if $A \subseteq B$ then $A^{\circ 5-g-c o c} \subseteq B^{\circ 5-g-c o c}$.

Proof :-

1. By Proposition (3.8) .

2. let $\mathrm{A}$ is $\mathfrak{5}$-g-coc-open, since $\mathrm{A}^{\circ \mathfrak{5}-\mathrm{g}-\operatorname{coc}}$ largest $\mathfrak{s}$-g-coc-open containing $\mathrm{A}$, then $\mathrm{A}^{\mathfrak{o s}-\mathrm{g}-\mathrm{coc}} \subseteq \mathrm{A}$ and since $\mathrm{A} \subseteq \mathrm{A}^{\circ \mathfrak{s}-\mathrm{g}-\mathrm{coc}}$, then $\mathrm{A}=\mathrm{A}^{\circ \mathfrak{s}-\mathrm{g}-\mathrm{coc}}$

Conversely; Let $A=A^{\circ \mathfrak{s}-g-c o c}$, since $A^{\circ \mathfrak{s}-g-c o c}$ is $\mathfrak{s}$-g-coc-open ,then $A$ is $\mathfrak{s}$-g-coc-open .

3. Let $x \in A^{\circ \mathfrak{s}}$ then there exists $U$ s-open set such that $x \in U \subseteq A$ then $U$ s-g-coc-open set then $U$ s-g-coc-open set such that $x \in U \subseteq A$ thus $x \in A^{\text {os-g-coc }}$

4. from (1) and (2).

5. Let $x \in A^{\circ \mathfrak{s}-g-\operatorname{coc}}$ then there exists $V \mathfrak{s}$-g-coc-open set such that $x \in V \subseteq A$ by proposition(3.19), since $A \subseteq B$ then $x \in V \subseteq B$. Then $x \in B^{\circ \mathfrak{s}-g-c o c}$ by proposition(3.14), Thus $A^{\circ \mathfrak{s}-g-c o c} \subseteq B^{\circ \mathfrak{s}-\mathrm{g}-c o c}$.

Definition (3.16)[3] Let $f: X \rightarrow Y$ be a function of space $X$ into space $Y$.Then $f$ is called supra-irresolute (scontinuous) function if $f^{-1}(A)$ is $\mathfrak{s}$-open set in $X$ for every s-open set $A$ in $Y$

Definition (3.17) Let $f: X \rightarrow Y$ be a function of a space $X$ into a space $Y$. $f$ is called $\mathfrak{s}$-g-coc-continuous function if $f^{-1}(A)$ is $\mathfrak{s}$-g-coc-open set in $X$ for every $\mathfrak{s}$-open set in $Y$.

Proposition (3.18) Every $\mathfrak{s}$-continuous is $\mathfrak{s}$-g-coc-continuous

\section{Proof}

Let $f: X \rightarrow Y$ be $\mathfrak{s}$-continuous function and $\mathrm{A}$ s-open set in $\mathrm{Y}$. Thus $f^{-1}(A)$ is $\mathfrak{s}$-open set in $\mathrm{X}$. Then $f^{-1}(A)$ is $\mathfrak{s - g}$-coc-open set in $\mathrm{X}$. Then $f$ is $\mathfrak{s}$-g-coc-continuous

But the convers not true in general for example

\section{Example (3.19)}

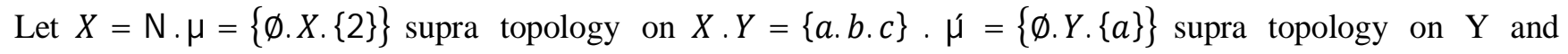
$f: X \rightarrow Y$ defined by $f(x)=\left\{\begin{array}{ll}a & \text { if } x \in\{1.3\} \\ b & \text { if } x \notin\{1.3\}\end{array}\right.$. Then $f$ is $\mathfrak{s}$-g-coc-continuous But not $\mathfrak{s}$-continuous

\section{Remark (3.20)}

Let $f: X \rightarrow Y$ be a function of a space $X$ in to a space $Y$ then

i. The constant function is $\mathfrak{s}$-g-coc-continuous

ii. If $(X, \mu)$ supra discrete topology then $f$ s-g-coc-continuous

iii. If $X$ finite set and $\mu$ any topology on $X$ then $f$ s-g-coc-continuous

iv. If $\left(\mathrm{Y}, \mu^{\prime}\right)$ indiscrete topology then $f$ s-g-coc-continuous

\section{Proposition (3.21)}

Let $f: X \rightarrow Y$ be a function of a space $X$ into a space $Y$ then the following statements are equivalent :-

1. $f$ s-g-coc-continuous function.

2. $f^{-1}\left(A^{\circ \mathfrak{S}}\right) \subseteq\left(f^{-1}(A)\right)^{\circ \mathfrak{o}-g-c o c}$ for every set $A$ of $Y$.

3. $f^{-1}(A) \mathfrak{s}$-g-coc-closed set in $X$ for every $\mathfrak{s}$-closed set $A$ in $X$. 
4. $f\left(\bar{A}^{\mathfrak{s}-\mathrm{g}-c o c}\right) \subseteq \overline{f(A)^{\mathfrak{s}}}$ for every set $A$ of $X$.

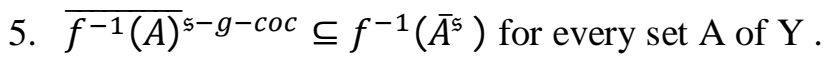

proof:

$1 \rightarrow 2$

Let $A \subseteq Y$, since $A^{\circ \mathfrak{s}} \quad \mathfrak{s}$-open set in $Y$.Then $f^{-1}\left(A^{\circ \mathfrak{5}}\right) \quad \mathfrak{s}$-g-coc-open set in $X$.Thus $f^{-1}\left(A^{\circ \mathfrak{5}}\right)=\left(f^{-1}\left(A^{\circ \mathfrak{5}}\right)\right)^{\circ \mathfrak{o}-g-\operatorname{coc}} \subseteq\left(f^{-1}(A)\right)^{\circ \mathfrak{o}-g-\operatorname{coc}}$. Hence $f^{-1}\left(A^{\circ \mathfrak{5}}\right) \subseteq(f(A))^{\circ \mathfrak{o}-g-\operatorname{coc}}$.

$2 \rightarrow 3$

Let $A \subseteq Y$ such that $A \mathfrak{s}$-closed set in $Y$.Then $A^{c}$ s-open set then $A^{c}=\left(A^{c}\right)^{\text {os }}$ then $f^{-1}\left(\left(A^{c}\right)^{\circ \mathfrak{s}}\right) \subseteq$ $\left(f^{-1}\left(A^{c}\right)\right)^{\circ \mathfrak{S}-g-c o c}$.Therefore $f^{-1}\left(A^{c}\right) \subseteq\left(f^{-1}\left(A^{c}\right)\right)^{\circ \mathfrak{S}-g-c o c}$ then $\left(f^{-1}(A)\right)^{c} \subseteq\left(f^{-1}(A)^{c}\right)^{\circ \mathfrak{S}-g-c o c}$. Therefore $\left(f^{-1}(A)\right)^{c}=\left(f^{-1}(A)^{c}\right)^{\text {os-g-coc }}$. Hence $\left(f^{-1}(A)\right)^{c}$ s-g-coc-open set in $X$. Hence $f^{-1}(A)$ s-g-cocclosed set in $X$.

$3 \rightarrow 4$

Let $A \subseteq X$. Then $\overline{f(A)^{\mathfrak{s}}}$ s-g-coc-closed set in $Y$.Then by (3) we have $f^{-1}\left(\overline{f(A)^{\mathfrak{s}}}\right)$ is $\mathfrak{s}$-g-coc-closed set in $X$ containing $A$,thus $\bar{A}^{\mathfrak{s}-g-c o c} \subseteq f^{-1}\left(\overline{f(A)^{\mathfrak{s}}}\right)$ ( since $\bar{A}^{\mathfrak{s}-\mathrm{g}-\operatorname{coc}}$

intersection of all $\mathfrak{s}$-g-coc-closed sets in $X$ containing A). Hence $f\left({\overline{(A)^{\mathfrak{s}}}}^{\mathrm{g}-\operatorname{coc}}\right) \subseteq \overline{f(A)^{\mathfrak{s}}}$

$4 \rightarrow 5$

Let $A \subseteq Y$.Then $f^{-1}(A) \subseteq X$.Then by (4) we have $f\left(\overline{\left(f^{-1}(A)\right)^{\mathfrak{s}}-g-c o c}\right) \subseteq \quad \overline{f\left(f^{-1}(A)\right)^{\mathfrak{s}}}$. Hence

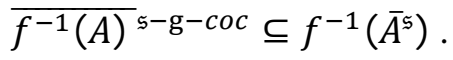

$5 \rightarrow 1$

Let $B \mathfrak{s}$-open set in $Y$ then $B^{c}$ s-closed.Then $B^{c}=\overline{B^{c^{\mathfrak{S}}}}$. Hence $\overline{f^{-1}\left(B^{c}\right)^{\mathfrak{s}}-\mathrm{g}-c o c} \subseteq f^{-1}\left(\overline{B^{c} \mathfrak{s}}\right)$. Then $\overline{f^{-1}\left(B^{c}\right)^{\mathfrak{s}}-g-c o c} \subseteq f^{-1}\left(B^{c}\right)$. Then $f^{-1}\left(B^{c}\right)=\left(f^{-1}(B)\right)^{c}$ s-g-coc-closed set in $X$. Therefore $f^{-1}(B)$ s-g-cocopen set in $\mathrm{X}$. Thus $f$ s-g-coc-continuous function .

Remark (3.22) From proposition (3.21) we have $f$ s-g-coc-continuous if and only if , the inverse image of every $\mathfrak{s}$-closed set in $Y$ is $\mathfrak{s}$-g-coc-closed set in $X$.

Definition (3.23) Let $f: X \rightarrow Y$ be a function of a space $X$ into a space $Y$, then $f$ is called 5 -g-coc irresolute $\left(\mathfrak{s}-g\right.$-coć-continuous ) function if $f^{-1}(A) \mathfrak{s}$-g-coc-open set in $X$ for every $\mathfrak{s}$-g-coc-open set in $Y$.

Proposition (3.24) Every $\mathfrak{s}-\mathrm{g}-$ coć-continuous function is $\mathfrak{s}$-g-coc-continuous function

Proof:

Let $f:(X . \mu) \rightarrow\left(Y . \mu^{\prime}\right)$ be $\mathfrak{s}-\mathrm{g}-$ coć - continuous and $B \mathfrak{s}$-open set in $Y$. Then $B$ is $\mathfrak{s}$-g-coc-open set. Since $f \mathfrak{s}-g$-coć-continuous then $f^{-1}(B)$ s-g-coc-open. Hence $f \mathfrak{s}$-g-coc-continuous function .

But the converse is not true in general for the following example.

Example(3.25): $\quad f: X \rightarrow Y$ be function defined by $f(x)=\left\{\begin{array}{ll}1 & \text { if } x \in \mathrm{Ne} \\ 2 & \text { if } x=\mathrm{x} 1 \\ 3 & \text { if } x \in \mathrm{No}\end{array}\right.$ and $\mathrm{X}=\mathrm{x}_{1} \cup \mathrm{N}\left(\mathrm{x}_{1} \in \mathrm{R}, \mathrm{N}\right.$ nature number,$\mu=\{\varnothing . X . A: A \subseteq N\} . \mu^{\prime}$ supra indiscrete topology on $Y=\{1.2 .3\}$, then the only s-open sets in $Y$ are Y and $\emptyset$, then $f^{-1}(\varnothing)=\emptyset, f^{-1}(Y)=X$. Since $Y$ and $\emptyset$ s-g-coc-open sets in $Y$ then $f^{-1}(\emptyset) . f^{-1}(Y) \mathfrak{s}$-g-coc- 
open in $X$.Then $f$ s-g-coc-continuous function. But $\{2\}$ s-g-coc-open in $Y$ and $f^{-1}(\{2\})=\{\mathrm{x} 1\}$ is not $\mathfrak{s}$-g-cocopen set in $X . \mathrm{x} 1 \in\{\mathrm{x} 1\}$. There is no $\mathfrak{s}$-g-open set $U$ such that $\mathrm{x} 1 \in U$, and $K$-compact such that $\mathrm{x} 1 \in U-$ $K \subseteq\{\mathrm{x} 1\}$. Then $f$ is not $\mathfrak{s}$-g-coc'-continuous function .

Proposition (3.26) Let $f: X \rightarrow Y$ be $\mathfrak{s}$-g-coc' -continuous then $f^{-1}(A)$ s-g-coc-closed set in $X$ for all A s-g-cocclosed set in Y

proof

Let $\mathrm{A}$ is $\mathfrak{s}$-g-coc-closed set in $\mathrm{Y}$.Then $A^{c}$ s-g-coc-open in $Y$. Since $f$-g-g-coc'-continuous . Then $f^{-1}\left(A^{c}\right)$ is $\mathfrak{s}$-gcoc-open in $X$ by definition(2.10). Since $f^{-1}\left(A^{c}\right)=\left(f^{-1}(A)\right)^{c}$. Then $\left(f^{-1}(A)\right)^{c} \mathfrak{s}$-g-coc-open set in $X$ .Therefore. $f^{-1}(A)$ s-g-coc-closed set in $X$ for all $A$ s-g-coc-closed set in $Y$.

\section{$\underline{\text { Remark (3.27) }}$}

i. $\quad \mathfrak{s}-g-\operatorname{coc}-$ continuous is need not to be $\mathfrak{s}$-continuous function .

ii. $\quad \mathfrak{s}$-continuous is need not to be $\mathfrak{s}-\mathrm{g}-$ coć-continuous function .

But the converse is not true as the follow example

\section{Examples (3.28)}

i--

Let $\quad f: X \rightarrow Y . X=\{1.2 .3\} \cdot \mu=\{\varnothing . X .\{3\}\}$ supra topology on $X \quad$ and $\quad Y=\{a . b\} \cdot \mu^{\prime}=$ $\{\varnothing . Y .\{a\}\}$ supra topology on $Y . f(1)=f(3)=b . f(2)=a$. It is clear that $\mathrm{f}$ is $\mathfrak{s}$-g-coć-continuous but not $\mathfrak{s}$-continuous .

ii- Let $f: X \rightarrow Y$ be function defined by $f(x)=\left\{\begin{array}{ll}1 & \text { if } x \in \mathrm{Ne} \\ 2 & \text { if } x=\mathrm{x} 1 \\ 3 & \text { if } x \in \mathrm{No}\end{array}\right.$ and $\mathrm{X}=\mathrm{x}_{1} \cup \mathrm{N}\left(\mathrm{x}_{1} \in \mathrm{R}, \mathrm{N}\right.$ natural number $), \mu$ $=\{\emptyset . X . A: A \subseteq N\} . \mu^{\prime}$ supra indiscrete topology on $Y=\{1.2 .3\}$, then the only s-open sets in $Y$ are $Y$ and $\emptyset$, then $f^{-1}(\varnothing)=\emptyset, f^{-1}(Y)=X$. Since $Y$ and $\emptyset$ s-open sets in $Y$ then $f^{-1}(\varnothing)$. $f^{-1}(Y)$ s-open in $X$.Then $f$ scontinuous function. But $\{2\}$ s-g-coc-open in $Y$ and $f^{-1}(\{2\})=\{\mathrm{x} 1\}$ is not s-g-coc-open set in $X . \mathrm{x} 1 \in\{\mathrm{x} 1\}$ . There is no $\mathfrak{s}$-g-open set $U$ such that $\mathrm{x} 1 \in U$, and $K$ s-compact such that $\mathrm{x} 1 \in U-K \subseteq\{\mathrm{x} 1\}$. Then $f$ is not $\mathfrak{s}$ g-coc'-continuous function .

\section{Proposition (3.29)}

Let $f: X \rightarrow Y$ be a function of space $X$ into space $Y$ then the following statements are equivalent .

i. $f$ is $\mathfrak{s}$-g-coć-continuous .

ii. $f\left(\bar{A}^{\mathfrak{s}-g-c o c}\right) \subseteq \overline{f(A)^{\mathfrak{s}}-g-\operatorname{coc}}$ for every set $A \subseteq X$.

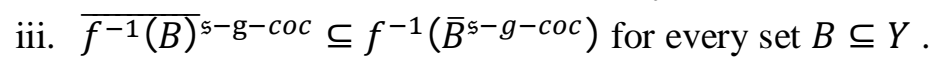

proof:

$i \rightarrow i i$

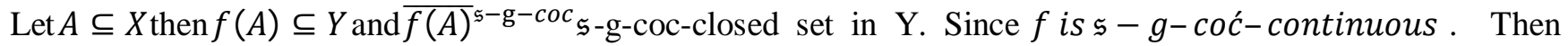

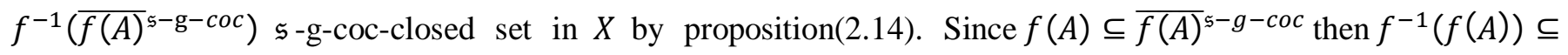

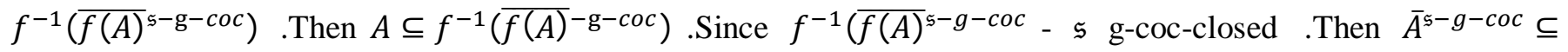
$f^{-1}\left(\overline{\left.f(A)^{\mathfrak{s}}-\mathrm{g}-\operatorname{coc}\right)}\right)$ thus $f\left(\bar{A}^{\mathfrak{s}-g-c o c}\right) \subseteq f\left(f^{-1}\left({\overline{f(A)^{\mathfrak{s}}}}^{-g-c o c}\right)\right) \subseteq \overline{f(A)^{\mathfrak{s}}-g-\operatorname{coc}}$. Then $f\left(\bar{A}^{\mathfrak{s}-g-\operatorname{coc}}\right) \subseteq \overline{f(A)^{\mathfrak{s}}-g-\operatorname{coc}}$ $i i \rightarrow i i i$

Let $\left(\bar{A}^{\mathfrak{s}-c o c}\right) \subseteq \overline{f(A)^{\mathfrak{s}-c o c}} \forall A \subseteq X . B \subseteq Y \quad$, then $\quad f^{-1}(B) \subseteq X, f\left(\overline{f^{-1}(B)^{\mathfrak{s}-c o c}}\right) \subseteq \overline{f\left(f^{-1}(B)\right)^{\mathfrak{s}-c o c}}$,then $f\left(\overline{f^{-1}(B)^{\mathfrak{s}}-\mathrm{g}-c o c}\right) \subseteq \bar{B}^{\mathfrak{s}-\mathrm{g}-c o c}$. Hence $\overline{f^{-1}(B)^{\mathfrak{s}}-g-c o c} \subseteq f^{-1}\left(\bar{B}^{\mathfrak{s}-\mathrm{g}-c o c}\right)$ 
$i i i \rightarrow i$

Let $B$ be $\mathfrak{s}$-g-coc-closed set in $Y$ then $\mathrm{B}=\bar{B}^{\mathfrak{s}-\mathrm{g}-c o c}$. Since $\overline{f^{-1}(B)^{\mathfrak{s}}-\mathrm{g}-\operatorname{coc}} \subseteq f^{-1}\left(\bar{B}^{\mathfrak{s}-\mathrm{g}-\operatorname{coc}}\right)$. Then $\overline{f^{-1}(B)^{\mathfrak{s}}-g-c o c} \subseteq f^{-1}(B)$. Since $f^{-1}(B) \subseteq \overline{f^{-1}(B)^{\mathfrak{s}}-g-c o c}$.Therefore $\overline{f^{-1}(B)^{\mathfrak{s}}-\mathrm{g}-c o c}=f^{-1}(B)$. Therefore $f^{-1}(B) \mathfrak{s}$-g-coc-closed in $\mathrm{X}$. Hence is $\mathfrak{s}-\mathrm{g}-$ coć - continuous .

Proposition (3.30) Let $f: X \rightarrow Y$ be a function of space $X$ into space $Y$ then $f$ is $\mathfrak{s}-\mathrm{g}$-coćcontinuous function if and only if the inverse image of every $\mathfrak{s}$-g-coc-closed in $Y$ is $\mathfrak{s}$-g-coc-closed set in $X$ proof:

Let $f$ be $\mathfrak{s}-\mathrm{g}$-có́c-continuous, let $B$ be $\mathfrak{s}$-g-coc-closed set in $Y$. Then $B^{c}$ s-g-coc-open in $Y$ Since $f \mathfrak{s}-$ g-coć-continuous, then $f^{-1}\left(B^{c}\right)$ s-g-coc-open in $X . f^{-1}\left(B^{c}\right)=f^{-1}(B-Y)=f^{-1}(Y)-f^{-1}(B)=X-$ $f^{-1}(B)=\left(f^{-1}(B)\right)^{c}$.Then $\left(f^{-1}(B)\right)^{c}$ s-g-coc-open in X. Hence $f^{-1}(B)$ s-g-coc-closed in $X$ Conversely:

Let $M$ be $\mathfrak{s}$-g-coc-open in $Y$, then $M^{c}$ is $\mathfrak{s}$-g-coc-closed in $Y$. Then $f^{-1}\left(M^{c}\right)$ s-g-coc-closed in $X$, since $f^{-1}\left(M^{c}\right)=\mathrm{f}^{-1}(\mathrm{Y}-\mathrm{M})=\mathrm{f}^{-1}(\mathrm{Y})-\mathrm{f}^{-1}(\mathrm{M})=\mathrm{X}-\mathrm{f}^{-1}(\mathrm{M})=\left(\mathrm{f}^{-1}(\mathrm{M})\right)^{\mathrm{c}}$. Therefore, $\mathrm{f}^{-1}\left(\mathrm{M}^{\mathrm{c}}\right)=\left(\mathrm{f}^{-1}(\mathrm{M})\right)^{\mathrm{c}}$.

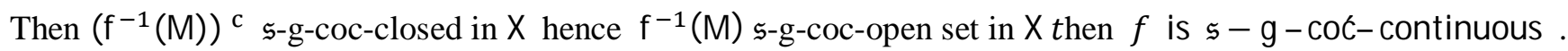

The following diagram shows the relation between types of $\mathfrak{s}$-coc-continuous functions

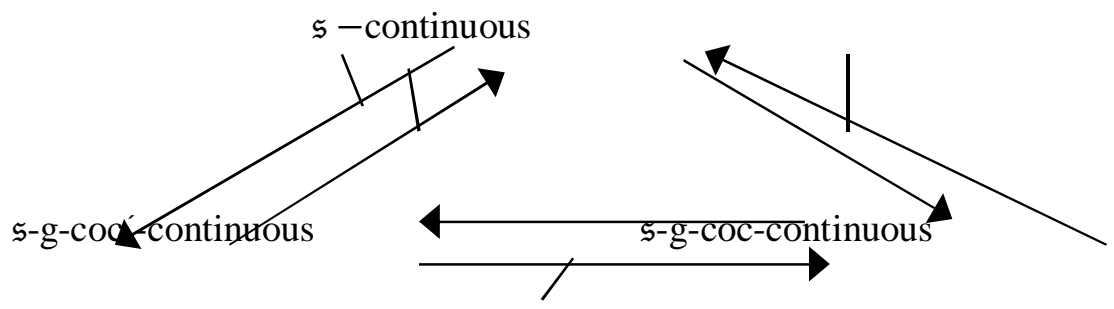

\section{4. s-g-Coc-compact space}

In this section, we introduce the concept of $\mathfrak{5}$-g-Coc-compact space and give some important generalization on this concept.

Definition (4.1): Let $X$ be a supra space. A family $F$ of subset of $X$ is called 5 -g-coc-open cover of $X$ if $F$ covers $X$ and $F$ is sub family of $\mu^{g k}$.

Definition (4.2): A space $X$ is said to be $\mathfrak{s}$-g-coc-compact if every $\mathfrak{s}$-g-coc-open cover of $X$ has finite sub cover.

\section{Example (4.3):}

.i. Every finite subset of a space $X$ is an $\mathfrak{s}$-g-coc-compact.

ii. The indiscrete space is $\mathfrak{5}$-g-coc-compact space.

Remark (4.4): It is clear that every $\mathfrak{s}$-g-coc-compact space is $\mathfrak{s}$-compact but the converse is not true in general as the following example shows 
Example (4.5): Let $X=\mathbb{R}$ the set of real numbers with $\tau$ is indiscrete topology, the coc-open set is $\{A: A \subseteq X\}$. Then $X$ is $\mathfrak{s}$-compact space but not $\mathfrak{s}-\mathrm{g}$-coc-copmpact.It is clear that, a space $(\mathrm{X} \mu)$ is $\mathfrak{s}$-g-coc-compact iff the space $\left(\mathrm{X} \mu^{g k}\right)$ is $\mathfrak{s}$-compact.

\section{Theorem (4.6):}

Let $f: X \rightarrow Y$ be an onto $\mathfrak{s}$-g-coc-continuous function. If $X$ is $\mathfrak{s}$-g-coc-compact then $Y$ is $\mathfrak{s}$-compact. Proof:

Let $\left\{V_{\alpha}: \alpha \in \Lambda\right\}$ be an $\mathfrak{5}$-open cover of $Y$ then $\left\{f^{-1}\left(V_{\alpha}\right): \alpha \in \Lambda\right\}$ is an $\mathfrak{5}$-g-coc-open cover of $X$, since $X$ is 5 -gcoc-compact. Then $X$ has finite sub cover say $\left\{f^{-1}\left(V_{\alpha i}\right): i=1.2 \ldots . . n\right\}$ and $V_{\alpha i} \in\left\{V_{\alpha}: \alpha \in \Lambda\right\}$. Hence $\left\{V_{\alpha i}: i=\right.$ $1.2 \ldots . . n\}$ is a finite sub cover of $Y$. Then $Y$ is $\mathfrak{s}$-compact.

\section{Theorem (4.7):}

Let $f: X \rightarrow Y$ be an onto $\mathfrak{s}-\mathrm{g}-\operatorname{coc}^{\prime}$-continuous function. If $X$ is $\mathfrak{s}$-g-coc-compact then $Y$ is $\mathfrak{s}$-g-coc-compact. Proof:

Let $\left\{V_{\alpha}: \alpha \in \Lambda\right\}$ be an $\mathfrak{s}$-g-coc-open cover of $Y$ then $\left\{f^{-1}\left(V_{\alpha}\right): \alpha \in \Lambda\right\}$ is an $\mathfrak{s}$-g-coc-open cover of $X$, since $X$ is s-g-coc-compact. Then $X$ has finite sub cover say $\left\{f^{-1}\left(V_{\alpha i}\right): i=1.2 \ldots . . n\right\}$ and $V_{\alpha i} \in\left\{V_{\alpha}: \alpha \in \Lambda\right\}$. Hence $\left\{V_{\alpha i}: i=1.2 \ldots . n\right\}$ is a finite sub cover of $Y$. Then $Y$ is $\mathfrak{s}$-g-coc-compact.

\section{Proposition (4.8):}

For any space $X$ the following statement are equivalent:

i. $X$ is $\mathfrak{s - g - c o c - c o m p a c t ~}$

ii. Every family of $\mathfrak{s}$-g-coc-closed sets $\left\{F_{\alpha}: \alpha \in \Lambda\right\}$ of $X$ such that $\bigcap_{\alpha \in \Lambda} F_{\alpha}=\phi$, then there exist a finite subset $\Lambda_{o} \subseteq \Lambda$ such that $\bigcap_{\alpha \in \Lambda} F_{\alpha}=\phi$.

Proof:

(i) $\rightarrow$ (ii) Assume that $X$ is $\mathfrak{s}$-g-coc-compact, let $\left\{F_{\alpha}: \alpha \in \Lambda\right\}$ be a family of $\mathfrak{s}$-g-coc-closed subset of $X$ such that $\bigcap_{\alpha \in \Lambda} F_{\alpha}=\phi$. Then the family $\left\{X-F_{\alpha}: \alpha \in \Lambda\right\}$ is $\mathfrak{s}$-g-coc-open cover of the $\mathfrak{s}$-g-coc-compact $(\mathrm{X}, \mu)$ there exist a finite subset $\Lambda_{o}$ of $\Lambda$ such that $X=\cup\left\{X-F_{\alpha}: \alpha \in \Lambda_{o}\right\}$ therefore $\phi=X-\cup\left\{X-F_{\alpha}: \alpha \in \Lambda_{o}\right\}=\cap\{X-(X-$ $\left.\left.F_{\alpha}\right): \alpha \in \Lambda_{o}\right\}=\cap\left\{X-F_{\alpha}: \alpha \in \Lambda_{o}\right\}$

(ii) $\rightarrow$ (i) Let $U=\left\{U_{\alpha}: \alpha \in \Lambda\right\}$ be an $\mathfrak{s}$-g-coc-open cover of the space $(X, \tau)$. Then $X-\left\{U_{\alpha}: \alpha \in \Lambda\right\}$ is a family of s-g-coc-closed subset of $(\mathrm{X}, \mu)$ with $\cap\left\{X-U_{\alpha}: \alpha \in \Lambda\right\}=\phi$ by assumption there exists a finite subset $\Lambda_{o}$ of $\Lambda$ such that $\left\{X-U_{\alpha}: \alpha \in \Lambda_{o}\right\}=\phi$ so $X=X-\cap\left\{X-U_{\alpha}: \alpha \in \Lambda_{o}\right\}=U\left\{U_{\alpha}: \alpha \in \Lambda_{o}\right\}$. Hence $X$ is $\mathfrak{s}$-g-coccompact.

\section{Definition (4.9):}

A subset $B$ of a space $X$ is said to be $\mathfrak{s}$-g-coc-compact relative to $X$ if for every cover of $B$ by $\mathfrak{s}$-g-coc-open sets of $X$ has finite sub cover of $B$. The sub set $B$ is $\mathfrak{s}$-g-coc-compact iff it is $\mathfrak{s}$-g-coc-compact as a sub space.

Proposition (4.10): If $X$ is a space such that every $\mathfrak{s}$-g-coc-open subset of $X$ is $\mathfrak{s}$-g-coc-compact relative to $X$, then every subset is $\mathfrak{5}$-g-coc-compact relative to $X$.

Proof:

Let $B$ be an arbitrary subset of $X$ and let $\left\{U_{\alpha}: \alpha \in \Lambda\right\}$ be a cover of $B$ by $\mathfrak{s}$-g-coc-open sets of $X$. Then the family $\left\{U_{\alpha}: \alpha \in \Lambda\right\}$ is a $\mathfrak{s}$-g-coc-open cover of the $\mathfrak{s}$-g-coc-open set $U\left\{U_{\alpha}: \alpha \in \Lambda\right\}$ Hence by hypothesis there is a finite subfamily $\left\{U_{\alpha i}: i=1.2 \ldots . . n\right\}$ which covers $\cup\left\{U_{\alpha}: \alpha \in \Lambda\right\}$. This the subfamily is also a cover of the set $B$.

Theorem (4.11): $\quad$ Every $\mathfrak{s}$-g-coc-closed subset of $\mathfrak{s}$-g-coc-compact space is $\mathfrak{s}$-g-coc-compact relative.

Proof: 
Let $A$ be an $\mathfrak{s - g - c o c - c l o s e d ~ s u b s e t ~ o f ~} X$. Let $\left\{U_{\alpha}: \alpha \in \Lambda\right\}$ be a cover of $A$ by $\mathfrak{s}$-g-coc-open subset of $X$. Now for each $x \in X-A$,there is a s-g-coc-open set $V_{x}$ such that $V_{x} \cap A$ is a finite. Since $X$ is $\mathfrak{s}$-g-coc-compact and the collection $\left\{U_{\alpha}: \alpha \in \Lambda\right\} \cup\left\{V_{x}: x \in X-A\right\}$ is a s-g-coc-open cover of $X$, there exists a finite sub cover $\left\{U_{\alpha i}: i=\right.$ $1 \ldots . . n\} \cup\left\{V_{x i}: i=1 \ldots . . n\right\}$.Since $\bigcup_{i=1}^{n}\left(V_{x i} \cap A\right)$ is finite, so for each $x_{j} \in\left(V_{x i} \cap A\right)$, there is $U_{\alpha\left(x_{j}\right)} \in$ $\left\{U_{\alpha}: \alpha \in \Lambda\right\}$ such that $x_{j} \in U_{\alpha\left(x_{j}\right)}$ and $j=1 \ldots \ldots$. Hence $\left\{U_{\alpha i}: i=1 \ldots \ldots n\right\}\left\{U_{\alpha\left(x_{j}\right)}: j=1 \ldots . . n\right\}$ is a finite sub cover of $\left\{U_{\alpha}: \alpha \in \Lambda\right\}$ and it covers $A$.Therefore, $A$ is $\mathfrak{s}$-g-coc-compact relative to $X$.

\section{Definition(4.12):}

i. A space $X$ is called $C C$ if every $\mathfrak{s}$-compact set in $X$ is $\mathfrak{s}$-closed.

ii. A space $X$ is called $C C^{\prime}$ if every $\mathfrak{5}$-g-coc-compact set in $X$ is $\mathfrak{s}$-g-coc-closed.

\section{Theorem(4.13):}

For any $\operatorname{space}(\mathrm{X}, \mu)$, then $\left(\mathrm{X} \mu^{g k}\right)$ is $\mathrm{CC}$.

Proof.

Let $\mathrm{K} \in \mathrm{C}\left(\mathrm{X} . \mu^{g k}\right)$. As $\mu \subseteq \mu^{g k}$, then $\mathrm{C}\left(\mathrm{X}, \mu^{g k}\right) \subseteq \mathrm{C}(\mathrm{X}, \mu)$ and hence $\mathrm{K} \in \mathrm{C}(\mathrm{X}, \mu)$. Thus, we have $\mathrm{X}-\mathrm{K} \in \mu^{g k}$, and hence $\mathrm{K}$ is $\mathfrak{s}$-closed in the space $\left(\mathrm{X}, \mu^{g k}\right)$.

\section{Theorem(4.14) :}

Let $X$ be a space. Then the following statements are equivalent:

i. $X$ is CC.

ii. $\mu=\mu^{g k}$.

Proof. i $\Rightarrow$ ii

Since $\mu \subseteq \mu^{g k}$,it is sufficient to see that $\mu^{g k} \subseteq \mu$, let $\mathrm{k}$ is $\mathfrak{s}$-compact then $\mathrm{X}-\mathrm{K} \in \mu^{g k}$, by $\mathrm{i}, \mathrm{X}-\mathrm{K} \in \mu$, then $\mu^{g k}=\mu$

ii $\Rightarrow \mathrm{i}$ Let $\mathrm{K} \in \mathrm{C}(\mathrm{X}, \tau)$. Then $\mathrm{X}-\mathrm{K} \in \mu^{g k}$, and by ii, $\mathrm{X}-\mathrm{K} \in \tau$. Therefore, $\mathrm{K}$ is $\mathfrak{s}$-closed in $\mathrm{X}$.

Remark(4.15): It is clear that every $C C$ space is $C C^{\prime}$ space but the converse is not true in general as the following example shows:

Example(4.16): Let $X=\{1.2 .3\}, \tau=\{X . \phi .\{1\} .\{2\} .\{1.2\}\}$, the $\mathfrak{s}$-coc-open sets are discrete. $\{1\}$ is $\mathfrak{s}$-compact set but not 5 -closed then $X$ is not $C C$ space.

\section{Definition (4.17):}

Let $f: X \rightarrow Y$ be a function of a space $X$ into a space $Y$ then $f$ is called 5 -g-coc-compact function if $f^{-1}(A)$ is $\mathfrak{5}$ compact set in $X$ for every s-coc-compact set $A$ in $Y$.

Remark (4.18): Every $\mathfrak{s}$-g- coc-compact function is $\mathfrak{s}$-compact function.

Definition (4.19): Let $f: X \rightarrow Y$ be a function of a space $X$ into a space $Y$ then $f$ is called $\mathfrak{s}-g-\operatorname{coc}^{\prime}$-compact function if $f^{-1}(A)$ is $\mathfrak{s}$-g-coc-compact set in $X$ for every $\mathfrak{s}$-g-coc-compact set $A$ in $Y$.

Example (4.20): Every function from a finite space into any space is $\mathfrak{s}-\mathrm{g}-\operatorname{coc}^{\prime}$-compact function.

Remark (4.21): Every $\mathfrak{s}-\mathrm{g}-\operatorname{coc}^{\prime}-$ compact function is $\mathfrak{s}$-g-coc-compact function.

\section{Remark (2.22) :}


Let $f: X \rightarrow Y$ be a function for which $X$ is CC then the following statements are equivalent:

i. $f$ is $\mathfrak{s - c o n t i n u o u s . ~}$

ii. $f$ is $\mathfrak{s - g - c o c - c o n t i n u o u s . ~}$

\section{Remark (2.23):}

Let $f: X \rightarrow Y$ be a function for which $X$ is $C C$ then the following statements are equivalent:

i. $f$ is $\mathfrak{s}$-continuous.

ii. $f$ is $\mathfrak{s}$-g- $\operatorname{coc}^{\prime}$-continuous.

\section{References}

[1] T. M. Al-shami, Some results related to supra topological spaces, Journal of Advanced Studies in Topology, 7 (4) (2016) 283-294.

[2] O. Ravi, G. Ramkumar and M. Kamaraj, On supra g-closed sets, International Journal of Advances in Pure and Applied Mathematics, 2 (2011),

no. $1,52-66$.

[3] A. S. Mashhour, A.A. Allam, F.S. Mahmoud and F.H. Khedr, On Supra topological spaces, Indian Jr. Pure and Appl. Math., 14 (1983), no. 4, 502-510.

[4] B. Meera Devi, D.K. Nathan R. Selvarathi, $\mu-\delta \mathrm{g}$-Continuous Functions in Supra Topological Spaces , Journal of Applied Science and Computations, 1076-5131 .

[5] O. R. SAYED , SUPRA PRE-OPEN SETS AND SUPRA PRE-CONTINUITY ON TOPOLOGICAL SPACES University of Bacau Faculty of Sciences Series Mathematics and Informatics Vol. 20 (2010), No. 2, 79 88 ,

[6] B.Meera Devi , New type of supra generalized closed set , Mathematical sciences international research journal : vol 3 sp1 Issue (2014) IssN 2349-1353.

[7] S. Al Ghour and S. Samarah " Cocompact Open Sets and Continuity", Abstract and Applied analysis, Volume 2012, Article ID 548612, 9 pages ,2012. 